Artículo

\title{
Modelos de predicción del desarrollo floral del aguacate 'Méndez'
}

\author{
Samuel Salazar-García ${ }^{1 \S}$ \\ Martha Elva Ibarra-Estrada ${ }^{2}$ \\ Arturo Álvarez-Bravo ${ }^{1}$ \\ José González-Valdivia \\ ${ }^{1}$ Campo Experimental Santiago Ixcuintla-INIFAP. Entronque carretera internacional México-Nogales km \\ 6, Santiago Ixcuintla, Nayarit, México. CP. 63 300. Tel. 01(55) 38718700, ext. 84426. ${ }^{2}$ Investigador(a) \\ independiente (marthaibarraes@hotmail.com; g.valdivia.jose@ hotmail.com). \\ ${ }^{\S}$ Autor para correspondencia: salazar.avocado@gmail.com.
}

\section{Resumen}

La disponibilidad de modelos de predicción brinda la oportunidad de pronosticar eventos importantes del desarrollo floral del aguacate. El objetivo de esta investigación fue desarrollar modelos de predicción, basados en temperatura, del desarrollo floral en aguacate 'Méndez'. La investigación se realizó en dos huertos comerciales de la empresa Agro González en el sur del estado de Jalisco, México. En cada huerto se eligieron 10 árboles y en cada uno de ellos se etiquetaron 30 brotes al inicio de cada flujo vegetativo (FV) de invierno (febrero 2014 y 2015) y verano (agosto 2014 y 2015). De cada árbol marcado se recolectó una yema apical mensual o quincenalmente según se aproximaba la fecha de antesis. Con el registro de las temperaturas mínimas (desde 8 a $20^{\circ} \mathrm{C}$ ) se calcularon los días frío (DF) y se acumularon para cada periodo de muestreo de yemas, denominándolos días frío acumulados (DFA). Mediante regresiones se identificaron las temperaturas asociadas al desarrollo floral y se obtuvieron modelos matemáticos de predicción. Posteriormente, se evaluó la capacidad para predecir el desarrollo floral de los mejores modelos de predicción obtenidos para cada FV en 2014 contra el mismo FV en 2015 y viceversa. Después de la verificación de la no diferencia entre años, se obtuvo un modelo de regresión para cada FV integrando la información de los dos años en un solo conjunto de datos. El desarrollo floral de yemas apicales de los FV de invierno y verano fue asociado a temperaturas $\leq 17$ (126 DFA) e $\leq 15{ }^{\circ} \mathrm{C}$ (138 DFA), respectivamente, y pudo ser modelado matemáticamente.

Palabras clave: Persea americana Miller, días frío, ecofisiología, floración.

Recibido: enero de 2018

Aceptado: febrero de 2018 


\section{Introducción}

En el aguacate (Persea americana Mill.), el tiempo transcurrido desde que los brotes vegetativos terminan su crecimiento e inician su desarrollo floral hasta antesis es variable y depende principalmente del cultivar y del clima en que se desarrolle (Salazar-García, 2002). En el sur de Florida el proceso de desarrollo floral del aguacate 'Choquette', desde que las yemas estaban en "reposo" (enero) hasta la máxima apertura floral (abril) cubrió tres meses. En el caso del cv. Booth-8 este mismo proceso requirió de cuatro meses (diciembre-abril) (Davenport, 1982).

En el clima mediterráneo semiárido del sur de California los brotes del flujo vegetativo de verano del cv. Hass necesitaron 7.5 meses desde la iniciación floral (agosto) hasta antesis (abril). El desarrollo de floral fue preliminarmente relacionado con temperaturas mínimas $\leq 15{ }^{\circ} \mathrm{C}$ (SalazarGarcía et al., 1998). En el clima semicálido subhúmedo del estado de Nayarit, México este mismo cultivar de aguacate requirió de casi 12 meses para que los brotes de invierno (emergidos en enero-febrero) desarrollaran hasta antesis (enero-febrero del siguiente año). Los brotes de verano, cinco meses más jóvenes que los de invierno, sólo ocuparon 7.5 meses para alcanzar antesis, de tal forma que la floración ocurrió en la misma fecha para los brotes florales de ambos flujos vegetativos.

Los promedios anuales de temperaturas máximas del aire fluctuaron de 26.8 a $33.4{ }^{\circ} \mathrm{C}$ y las mínimas de 9.3 a $20.4{ }^{\circ} \mathrm{C}$ (Salazar-García et al., 2007). En un estudio realizado en seis tipos de clima del estado de Michoacán, México el proceso de desarrollo floral en brotes de los flujos de invierno, primavera y verano, que produjeron floración normal (principal), el tiempo transcurrido fue de: 13 a 13.5, 9.5 a 10.5 y 7 a 7.5 meses, respectivamente. En todos los climas las temperaturas medias del aire variaron de $14{ }^{\circ} \mathrm{C}$ a $24{ }^{\circ} \mathrm{C}$ (Rocha-Arroyo et al., 2011).

El agobio hídrico no ha sido eficaz para promover la floración del aguacate 'Hass'. Estudios realizados en condiciones controladas mostraron que el único factor asociado con la iniciación floral del aguacate fue el descenso de la temperatura (Chaikiattiyos et al., 1994). En aguacate 'Fuerte' se encontró que la ausencia de altas temperaturas $\left(\geq 20^{\circ} \mathrm{C}\right)$, más que la presencia de bajas temperaturas, fue responsable de la iniciación floral (Buttrose y Alexander, 1978). En otro estudio en cámaras de crecimiento, Nevin y Lovatt (1989) promovieron la floración de árboles jóvenes de 'Hass' con estrés de baja temperatura $\left(8 \mathrm{~h}\right.$ día de $15{ }^{\circ} \mathrm{C}$ a $18{ }^{\circ} \mathrm{C}$ y $16 \mathrm{~h}$ noche de $10{ }^{\circ} \mathrm{C}$ a $\left.13{ }^{\circ} \mathrm{C}\right)$.

Al final de cuatro u ocho semanas los árboles fueron transferidos al invernadero con temperatura aproximada de $24^{\circ} \mathrm{C}, 12$-h día y $19^{\circ} \mathrm{C}, 12$-h noche. La intensidad de floración fue la misma para las plantas tratadas con cuatro u ocho semanas de baja temperatura y el desarrollo de los brotes florales resultó similar en tiempo, a excepción de la fecha de antesis, la cual ocurrió una semana después en los árboles que recibieron ocho semanas de baja temperatura. Los árboles testigo $\left(24^{\circ} \mathrm{C}, 12\right.$-h día y $19^{\circ} \mathrm{C}, 12$-h noche, durante todo el experimento) no florecieron. En otro estudio en cámaras de crecimiento, Salazar-García et al. (1999) obtuvieron resultados similares ya que después de cuatro semanas de estrés por baja temperatura $\left(\leq 15^{\circ} \mathrm{C}\right)$ las yemas apicales de árboles jóvenes de 'Hass' alcanzaron la determinación irreversible hacia floración (DIF). Un trabajo posterior realizado en huertos adultos de 'Hass' en Nayarit, México se obtuvo una asociación entre temperaturas $\leq 19^{\circ} \mathrm{C}$ y la DIF (Salazar-García et al., 2006). 
Los modelos de predicción que simulan el crecimiento y desarrollo de los cultivos son herramientas importantes para la investigación agrícola moderna ya que permiten la representación sencilla de los procesos fisiológicos mediante ecuaciones matemáticas (López-Cruz et al., 2005). Dado que el desarrollo floral del aguacate ha sido asociado verazmente con la temperatura se han desarrollado modelos de predicción para varias regiones de México. En el clima semicálido de Nayarit se asoció el proceso de desarrollo floral del cv. Hass con la acumulación de días frío (DFA) con temperaturas mínimas $\leq 21 \mathrm{y} \leq 19-20{ }^{\circ} \mathrm{C}$; esto para brotes de los flujos vegetativos de invierno y verano, respectivamente (Salazar-García et al., 2007).

Posteriormente, estos modelos de predicción fueron probados en cuatro tipos de clima de la franja aguacatera de Michoacán (semicálido subhúmedo seco, semicálido subhúmedo, templado subhúmedo y templado húmedo). De todos los modelos de predicción desarrollados en Nayarit, sólo el modelo para brotes de verano DFA $\leq 19{ }^{\circ} \mathrm{C}$ presentó una elevada capacidad de predicción $\left(\mathrm{R}^{2}=0.94\right)$ del desarrollo floral en brotes de verano en los cuatro climas de Michoacán en donde se presentó este flujo vegetativo (Salazar-García et al., 2009).

La disponibilidad de modelos de predicción brinda la oportunidad de pronosticar eventos importantes o alteraciones del desarrollo floral del aguacate como la DIF, estado coliflor (elongación de ejes secundarios de la inflorescencia, la meiosis ha ocurrido en los lóculos de la antera y las microesporas son evidentes) o antesis (el estigma está receptivo y el polen puede ser liberado) (Salazar-García et al., 1998). Con esto se puede tener sustento para la planeación y toma de decisiones sobre algunas prácticas de manejo del huerto, como la introducción de abejas polinizadoras, la poda, control de plagas y enfermedades que se presentan durante la floración, fertilizantes y biorreguladores autorizados para así aumentar su efecto y su relación beneficio-costo.

El aguacate 'Méndez No. 1' (Plant Patent 11173 USA 2002), conocido en México como 'Méndez', tiene importancia económica en el sur del estado de Jalisco, donde hay más de 6000 ha plantadas. En esta región su principal cosecha anual ocurre en el verano pudiendo alcanzar los precios más altos del año, diferente a la de 'Hass' que se realiza en otoño-invierno. No se dispone de información sobre el proceso de desarrollo floral de 'Méndez' por lo que el objetivo de esta investigación fue desarrollar modelos de predicción, basados en temperatura, del desarrollo floral en aguacate 'Méndez'.

\section{Materiales y métodos}

\section{Características de los huertos}

La investigación se realizó durante dos ciclos de producción (2014-2015 y 2015-2016) en dos huertos comerciales de aguacate 'Méndez' injertados sobre portainjertos criollos de la empresa Agro González, SPR de RL, con fertirriego, suelo Feozem háplico y clima semicálido subhúmedo [AC(w)] (García-Amaro, 1998) del sur del estado de Jalisco. El huerto "Colorín 1" se ubica en Atequizayan, Municipio de Zapotlán el Grande y el huerto "Ocote cuate 2" está en Zapotiltic, Municipio de Zapotiltic. Para estos huertos, la altitud es $1556 \mathrm{~m}$ y $1428 \mathrm{~m}$, el distanciamiento entre árboles $7 \times 3.5 \mathrm{~m}$ y $5 \times 5 \mathrm{~m}$, la edad al inicio del estudio de 4 y 6 años, respectivamente. Los huertos recibieron el manejo estándar del productor. 


\section{Muestreo y procesamiento de yemas}

En cada huerto fueron seleccionados 10 árboles sin entrecruzamiento de copas y con historial de producción de al menos $50 \mathrm{~kg}$ árbol ${ }^{-1}$. A cada árbol se le marcaron 30 brotes al inicio de cada flujo vegetativo de invierno (febrero 2014 y 2015) y verano (agosto 2014 y 2015). De cada árbol marcado se recolectó una yema apical a intervalos mensuales o quincenales según se aproximaba la antesis. Para el flujo vegetativo de invierno (ambos años) los muestreos se realizaron de febrero a octubre y para el de verano de agosto a marzo del siguiente año.

Las yemas fueron fijadas en FAA (formaldehído:ácido acético:etanol, 5:5:90, v:v:v) y después introducidas a una campana de vacío (Nalgene 8040317, Nalgen Company) a $30 \mathrm{KPa}$ por 5 h. Posteriormente, fueron clasificadas bajo un microscopio estereoscópico (Zeiss Stereomikroskop Mod. Stemi 2000-C, Carl Zeiss, Göttingen, Germany), con la escala visual de Salazar-García et al. (1998) la cual comprende desde E-1 (yema vegetativa) a E-11 (antesis).

\section{Cálculo de días frío (DF)}

En cada huerto fue registrada cada $15 \mathrm{~min}$ la temperatura del aire con registradores automatizados HOBO H8 (Onset Computer, Witzprod, Englewood Cliffs, NJ, USA) operados con baterías. De forma independiente para brotes de los flujos vegetativos de invierno y verano se cuantificó la ocurrencia de temperaturas mínimas desde 8 a $20{ }^{\circ} \mathrm{C}$, en incrementos de $1{ }^{\circ} \mathrm{C}$. Se empleó la fórmula: $\mathrm{DF}=($ Tmín $\leq \mathrm{T}, 1,0)$; donde: Tmín= temperatura mínima registrada; $\mathrm{T}=$ temperatura crítica, de 8 a $20{ }^{\circ} \mathrm{C}$. Si se cumple la condición de temperatura, entonces el valor de DF es 1, en caso contrario es 0 . Mediante el programa Excel (2010). Los valores de $\mathrm{DF}_{8}$ hasta $\mathrm{DF}_{20}$, se acumularon para cada periodo de muestreo de yemas apicales. A estos valores se les denominó días frío acumulados (DFA). Como día cero se consideró cuando las yemas apicales estaban en E-1 (etapa vegetativa), lo que ocurrió en febrero (brotes de invierno) y agosto (brotes de verano).

\section{Identificación de la temperatura asociada con el desarrollo floral}

Los DFA se usaron como variables independientes y el estado de desarrollo floral de las yemas apicales en brotes de cada flujo como variable dependiente. Se usó el procedimiento Stepwise SAS/STAT (SAS, 2011) para seleccionar el mejor modelo por orden de respuesta (segundo hasta quinto orden) para cada temperatura seleccionada ( $\mathrm{de} \leq 8{ }^{\circ} \mathrm{C}$ hasta $\leq 20{ }^{\circ} \mathrm{C}$, en intervalos de $1{ }^{\circ} \mathrm{C}$ ). Los criterios para elegir los mejores modelos de predicción fueron: 1) mayor valor de $\mathrm{R}^{2} ; 2$ ) menor cuadrado medio del error (CME); 3) el valor de Cp (Draper y Smith, 1981).

Una vez identificados los mejores modelos de predicción, se calcularon sus coeficientes matemáticos $\left(\mathrm{B}_{0}, \ldots \ldots ., \mathrm{B}_{\mathrm{n}}\right)$ por el procedimiento REG utilizando los DFA, a partir de $\leq 8{ }^{\circ} \mathrm{C}$ hasta $\leq 20^{\circ} \mathrm{C}$, en intervalos de $1{ }^{\circ} \mathrm{C}$.

\section{Prueba de los mejores modelos de predicción}

Se evaluó la capacidad para predecir el desarrollo floral de los modelos de predicción obtenidos para cada flujo vegetativo en el año 1 contra el mismo flujo vegetativo en el año 2 y viceversa. Los valores de desarrollo floral predichos fueron analizados mediante una regresión contra los valores observados del desarrollo floral del año y flujo vegetativo correspondiente, utilizando el programa 
Excel (2010). Los criterios para determinar si los valores de los dos años pertenecían a una sola población fueron: 1) que la ordenada al origen de la regresión fuera cercano a uno $\left(\mathrm{B}_{0}=1\right)$; 2) que la pendiente fuera lo más aproximada a uno $\left(\mathrm{B}_{1}=1\right)$; y 3$)$ el mayor valor del coeficiente del modelo ajustado $\left(\mathrm{R}^{2}\right)$. Este procedimiento sirvió para depurar modelos y encontrar el mejor.

Posterior a la verificación de la no diferencia entre años, se obtuvo un modelo de regresión para cada flujo vegetativo integrando la información de los dos años en un solo conjunto de datos.

\section{Resultados y discusión}

\section{Desarrollo floral}

El estado de desarrollo floral de las yemas apicales de brotes de los flujos vegetativos de invierno y verano, fueron utilizados para generar los modelos de predicción del desarrollo floral (Cuadro 1 y 2). En promedio, el desarrollo floral (yema vegetativa a antesis) en brotes del flujo vegetativo de invierno y verano tardó 216 y 187 días, respectivamente. El menor tiempo requerido para que los brotes de verano de 'Méndez' completaran su desarrollo floral pudo deberse a que cuando éstos emergieron (agosto-septiembre) la temperatura ambiental ya había iniciado su descenso (promedio de $18{ }^{\circ} \mathrm{C}$ ), reprimiendo el crecimiento vegetativo y estimulando el desarrollo floral.

Cuadro 1. Estado de desarrollo floral de brotes del flujo vegetativo de invierno 2014 y 2015 en huertos de aguacate 'Méndez' empleados en la investigación.

\begin{tabular}{|c|c|c|c|}
\hline \multicolumn{2}{|c|}{ Huerto "Colorín 1" } & \multicolumn{2}{|c|}{ Huerto "Ocote Cuate 2" } \\
\hline Fecha & Desarrollo floral $^{\mathrm{Z}}$ & Fecha & Desarrollo floral $^{\mathrm{Z}}$ \\
\hline \multicolumn{4}{|c|}{ Flujo vegetativo de invierno 2014} \\
\hline 27 febrero 2014 & 1.1 & 27 febrero 2014 & 1.1 \\
\hline 29 marzo & 2 & 29 marzo & 2 \\
\hline 28 abril & 3.4 & 28 abril & 3.2 \\
\hline 28 mayo & 4.6 & 28 mayo & 4.5 \\
\hline 27 junio & 4.8 & 27 junio & 4.9 \\
\hline 27 julio & 6.1 & 27 julio & 5.5 \\
\hline 11 agosto & 7.8 & 26 agosto & 7.2 \\
\hline 25 agosto & 8.7 & 10 septiembre & 8.7 \\
\hline 10 septiembre & 10.6 & 25 septiembre & 10.1 \\
\hline 25 septiembre & 11 & 10 octubre & 11 \\
\hline \multicolumn{4}{|c|}{ Flujo vegetativo de invierno 2015} \\
\hline 25 febrero 2015 & 1.3 & 26 febrero 2015 & 1.3 \\
\hline 27 marzo & 2.3 & 27 marzo & 2.5 \\
\hline 26 abril & 3.6 & 26 abril & 3.4 \\
\hline 26 mayo & 4.4 & 26 mayo & 4.3 \\
\hline 25 junio & 4.7 & 25 junio & 4.5 \\
\hline 25 julio & 5.3 & 25 julio & 5.7 \\
\hline 24 agosto & 6.5 & 24 agosto & 6.3 \\
\hline 08 septiembre & 10.2 & 08 septiembre & 7.8 \\
\hline \multirow[t]{2}{*}{23 septiembre } & 11 & 23 septiembre & 10.7 \\
\hline & & 08 octubre & 11 \\
\hline
\end{tabular}

${ }^{z}=$ de acuerdo con la escala visual de Salazar-García et al. (1998). 
Cuadro 2. Estado de desarrollo floral de brotes del flujo vegetativo de verano 2014 y 2015 en huertos de aguacate 'Méndez' empleados en la investigación.

\begin{tabular}{|c|c|c|c|}
\hline \multicolumn{2}{|c|}{ Huerto "Colorín 1" } & \multicolumn{2}{|c|}{ Huerto "Ocote Cuate 2" } \\
\hline Fecha & Desarrollo floral $^{\mathrm{z}}$ & Fecha & Desarrollo floral $^{\mathrm{z}}$ \\
\hline \multicolumn{4}{|c|}{ Flujo vegetativo de verano 2014} \\
\hline 26 agosto 2014 & 1.1 & 26 agosto 2014 & 1.1 \\
\hline 25 septiembre & 2.6 & 25 septiembre & 2.4 \\
\hline 26 octubre & 3.9 & 26 octubre & 3.2 \\
\hline 25 noviembre & 6.1 & 25 noviembre & 4.5 \\
\hline 28 diciembre & 7.9 & 28 diciembre & 5.6 \\
\hline 24 enero & 9.7 & 24 enero & 8.6 \\
\hline 23 febrero & 11 & 23 febrero & 11 \\
\hline \multicolumn{4}{|c|}{ Flujo vegetativo de verano 2015} \\
\hline 24 agosto 2015 & 1.1 & 24 agosto 2015 & 1.1 \\
\hline 23 septiembre & 2 & 23 septiembre & 2.1 \\
\hline 26 octubre & 2.5 & 26 octubre & 2.5 \\
\hline 26 noviembre & 3.2 & 26 noviembre & 3 \\
\hline 25 diciembre & 5.8 & 25 diciembre & 4.8 \\
\hline 27 enero 2016 & 6.5 & 27 enero 2016 & 6.5 \\
\hline 11 febrero & 7.3 & 11 febrero & 8.2 \\
\hline 18 febrero & 8.5 & 18 febrero & 9.7 \\
\hline 26 febrero & 10.1 & 26 febrero & 10.5 \\
\hline 04 marzo & 11 & 04 marzo & 11 \\
\hline
\end{tabular}

${ }^{z}=$ de acuerdo con la escala visual de Salazar-García et al. (1998).

\section{Efecto de la temperatura ambiental sobre el desarrollo floral}

De todas las temperaturas mínimas utilizadas $\left(\leq 8{ }^{\circ} \mathrm{C}\right.$ hasta $\left.\leq 20{ }^{\circ} \mathrm{C}\right)$, las $\leq 17 \mathrm{y} \leq 15{ }^{\circ} \mathrm{C}$ fueron las que mejor se asociaron con el desarrollo floral en brotes de los flujos vegetativos de invierno y verano (dos años en cada caso), respectivamente (Cuadro 3 y 4). Buttrose y Alexander (1978); Salazar-García et al. (1999) encontraron en árboles jóvenes de 'Fuerte' y 'Hass', respectivamente, que en condiciones controladas las temperaturas constantes $>20{ }^{\circ} \mathrm{C}$ no promovieron la floración.

Posteriormente, en un estudio de campo con aguacate 'Hass' en Nayarit, México, Salazar-García et al. (2007) asociaron las temperaturas $\leq 21{ }^{\circ} \mathrm{C}$ al desarrollo floral de brotes del flujo de invierno y las $\leq 19$ y $\leq 20^{\circ} \mathrm{C}$ al de brotes de verano. En una investigación realizada en Michoacán (clima semicálido subhúmedo) se encontró que las temperaturas de $\leq 19$ hasta $\leq 21{ }^{\circ} \mathrm{C}$ se relacionaron con el proceso de desarrollo floral en brotes de los flujos de invierno y verano del cv. Hass (Salazar-García et al., 2009).

En el presente estudio, el proceso de desarrollo floral desde yemas en estado E-1 (término de alargamiento del brote vegetativo) hasta antesis (E-11) en brotes de invierno para 2014 y 2015 acumuló 111 y $140 \mathrm{DFA}\left(\leq 17^{\circ} \mathrm{C}\right)$, respectivamente. En brotes de verano se acumularon 133 
DFA (2014) y 143 DFA (2015) con temperaturas $\leq 15{ }^{\circ} \mathrm{C}$. En los dos años del estudio se acumularon en promedio 126 y 138 días con temperaturas $\leq 17 \mathrm{y} \leq 15{ }^{\circ} \mathrm{C}$ para brotes de invierno y verano, respectivamente.

Cuadro 3. Modelos seleccionados de predicción del desarrollo floral en brotes de los flujos vegetativos de invierno 2014 y 2015, que florecieron en verano 2014 y 2015 , respectivamente, en árboles de aguacate 'Méndez'.

\begin{tabular}{|c|c|c|c|c|c|c|c|c|c|c|}
\hline Temperatura & Bo & $\mathrm{B}_{1}$ & $\mathrm{~B}_{2}$ & $\mathrm{~B}_{3}$ & $\mathrm{~B}_{4}$ & $\mathrm{~B}_{5}$ & $\mathrm{~B}_{6}$ & $\mathrm{R}^{2}$ & $\mathrm{Cp}$ & CME \\
\hline \multicolumn{11}{|c|}{ Parámetros (brotes de invierno 2014) } \\
\hline$\leq 15^{\circ} \mathrm{C}$ & 1.51 & & & $-6.62 \mathrm{E}-05$ & $2.65 \mathrm{E}-06$ & $-2.92 \mathrm{E}-08$ & $9.92 \mathrm{E}-11$ & 0.76 & 4.66 & 3.07 \\
\hline$\leq 16^{\circ} \mathrm{C}$ & 1.55 & & & 7.13E-06 & $-3.42 \mathrm{E}-08$ & & -13 & 0.9 & 2.83 & 1.24 \\
\hline$\leq 17^{\circ} \mathrm{C}^{\mathrm{z}}$ & 1.13 & 0.006 & & 2.77E-05 & $-4.75 \mathrm{E}-07$ & 2.83E-09 & $-5.59 \mathrm{E}-12$ & 0.99 & 5.13 & 0.13 \\
\hline$\leq 18^{\circ} \mathrm{C}$ & 1.04 & & 0.001 & $-1.29 \mathrm{E}-05$ & & $4.243 \mathrm{E}-10$ & $-1.19 \mathrm{E}-12$ & 0.95 & 4.07 & 0.65 \\
\hline$\leq 19^{\circ} \mathrm{C}$ & 1.05 & & 0.001 & $-1.26 \mathrm{E}-05$ & & 4.10E-10 & $-1.15 \mathrm{E}-12$ & 0.95 & 4.08 & 0.69 \\
\hline \multicolumn{11}{|c|}{ Parámetros (brotes de invierno 2015) } \\
\hline$\leq 15^{\circ} \mathrm{C}$ & -2.4 & 0.227 & & $-1.58 \mathrm{E}-04$ & $3.08 \mathrm{E}-06$ & $-2.15 \mathrm{E}-08$ & 5.1 & 0.8 & 4.79 & 2.59 \\
\hline$\leq 16{ }^{\circ} \mathrm{C}$ & 0.71 & 0.00184 & 0.00158 & $-2.57 \mathrm{E}-05$ & $1.65 \mathrm{E}-07$ & $-3.51 \mathrm{E}-10$ & & 0.89 & 3.97 & 1.51 \\
\hline$\leq 17^{\circ} \mathrm{C}^{\mathrm{Z}}$ & 1.67 & -0.0245 & & $2.89 \mathrm{E}-05$ & $-4.12 \mathrm{E}-07$ & $2.08 \mathrm{E}-09$ & $-3.53 \mathrm{E}-12$ & 0.98 & 4.21 & 0.33 \\
\hline$\leq 18^{\circ} \mathrm{C}$ & 1.51 & -0.015 & & $2.25 \mathrm{E}-05$ & $-3.05 \mathrm{E}-07$ & $1.45 \mathrm{E}-09$ & $-2.31 \mathrm{E}-12$ & 0.98 & 4.1 & 0.36 \\
\hline$\leq 19^{\circ} \mathrm{C}$ & 1.51 & -0.0146 & & $2.23 \mathrm{E}-05$ & $-3.02 \mathrm{E}-07$ & $1.43 \mathrm{E}-09$ & $-2.28 \mathrm{E}-12$ & 0.97 & 3.03 & 0.39 \\
\hline
\end{tabular}

${ }^{\mathrm{z}}=$ mejores modelos.

Cuadro 4. Modelos seleccionados de predicción del desarrollo floral en brotes de los flujos vegetativos de verano 2014 y 2015, que florecieron en invierno 2015 y 2016, respectivamente, en árboles de aguacate 'Méndez'.

\begin{tabular}{|c|c|c|c|c|c|c|c|c|c|c|}
\hline Temperatura & Bo & $\mathrm{B}_{1}$ & $\mathrm{~B}_{2}$ & $\mathrm{~B}_{3}$ & $\mathrm{~B}_{4}$ & $\mathrm{~B}_{5}$ & $\mathrm{~B}_{6}$ & $\mathrm{R}^{2}$ & $\mathrm{Cp}$ & CME \\
\hline \multicolumn{11}{|c|}{ Parámetros (brotes de verano 2014) } \\
\hline$\leq 13^{\circ} \mathrm{C}$ & 2.06 & 0.3 & -0.01 & $1.44 \mathrm{E}-04$ & & $-1.19 \mathrm{E}-08$ & $5.49 \mathrm{E}-11$ & 0.96 & 6 & 0.78 \\
\hline$\leq 14^{\circ} \mathrm{C}$ & 1.84 & 0.18 & -0.004 & 3.93E-05 & $-1.43 \mathrm{E}-07$ & & & 0.98 & 4.6 & 0.35 \\
\hline$\leq 15^{\circ} \mathrm{C}^{\mathrm{z}}$ & 1.6 & 0.11 & -0.001 & $1.05 \mathrm{E}-05$ & $-1.82 \mathrm{E}-10$ & & & 0.98 & 3.02 & 0.31 \\
\hline$\leq 16^{\circ} \mathrm{C}$ & 1.71 & & & 4.63E-05 & $-8.52 \mathrm{E}-07$ & $5.76 \mathrm{E}-09$ & $-1.34 \mathrm{E}-11$ & 0.98 & 5.28 & 0.44 \\
\hline$\leq 17^{\circ} \mathrm{C}$ & 0.08 & 0.05 & & & $-1.56 \mathrm{E}-08$ & $2 \mathrm{E}-10$ & $-5.86 \mathrm{E}-13$ & 0.97 & 4 & 0.47 \\
\hline \multicolumn{11}{|c|}{ Parámetros (brotes de verano 2015) } \\
\hline$\leq 13^{\circ} \mathrm{C}$ & 1.44 & 0.0559 & & $-2 \mathrm{E}-04$ & $9.67 \mathrm{E}-06$ & $-1.41 \mathrm{E}-07$ & $6.49 \mathrm{E}-10$ & 0.835 & 4.27 & 2.62 \\
\hline$\leq 14^{\circ} \mathrm{C}$ & 1.33 & 0.0113 & & $3.3 \mathrm{E}-05$ & $-9.33 \mathrm{E}-07$ & $9.58 \mathrm{E}-09$ & $-3.20 \mathrm{E}-11$ & 0.882 & 3.99 & 1.88 \\
\hline$\leq 15^{\circ} \mathrm{C}^{\mathrm{z}}$ & 1.51 & 0.005 & 0.0011 & & $-5.95 \mathrm{E}-07$ & $1.07 \mathrm{E}-08$ & $-5.09 \mathrm{E}-11$ & 0.943 & 4 & 0.91 \\
\hline$\leq 16^{\circ} \mathrm{C}$ & -1.65 & 0.1973 & -0.0038 & $3.21 \mathrm{E}-05$ & $-8.23 \mathrm{E}-08$ & & & 0.915 & 4.24 & 1.34 \\
\hline$\leq 17^{\circ} \mathrm{C}$ & -0.82 & 0.1123 & -0.0016 & $9.13 \mathrm{E}-06$ & & $-5.98 \mathrm{E}-11$ & & 0.968 & 4 & 0.51 \\
\hline
\end{tabular}

${ }^{\mathrm{z}}=$ mejores modelos.

La cantidad de frío acumulado por las yemas apicales de los brotes de ambos flujos vegetativos fue menor que la encontrada para 'Hass' en el clima semicálido subhúmedo de Nayarit para brotes de invierno (179 DFA con temperaturas $\left.\leq 21{ }^{\circ} \mathrm{C}\right)$ y verano (183 y 201 DFA con 
temperaturas $\leq 19$ y $\leq 20{ }^{\circ} \mathrm{C}$, respectivamente) (Salazar-García et al., 2007). En Michoacán, para este mismo cultivar y tipo de clima se requirieron 375 (invierno) y 198 (verano) DFA con temperaturas $\leq 19 \mathrm{y} \leq 21{ }^{\circ} \mathrm{C}$ (Salazar-García et al., 2009). Las diferencias descritas muestran la plasticidad genética del aguacate a diferentes ambientes en los que se le cultiva y la aparente irrelevancia de establecer un valor único o crítico de temperatura asociada al desarrollo floral ya que el "frío" necesario para iniciar y completar un ciclo de desarrollo floral depende de las temperaturas que ocurren en cada región productora.

\section{Verificación de la no diferencia entre año 1 (2014) vs año 2 (2015)}

La verificación de los modelos de predicción del desarrollo floral (ajuste de valores observados $v s$. predichos) fue realizada en forma recíproca (año $1 v s$. año 2 y viceversa), para determinar si los datos de los dos años eran estadísticamente iguales (Figuras 1A-D). Para brotes de invierno el modelo DFA $\leq 17{ }^{\circ} \mathrm{C}$ del año 2 mostró una alta capacidad de predicción del desarrollo floral para el año $1\left(\mathrm{R}^{2}=0.99\right)$ (Figura 1A). Algo similar se obtuvo al usar el modelo del año 1 para predecir el desarrollo floral en el año $2\left(\mathrm{R}^{2}=0.99\right)$ (Figura 1B). Para brotes de verano se probó el modelo DFA $\leq 15{ }^{\circ} \mathrm{C}$ del año 2 el cual mostró una buena capacidad de predicción del desarrollo floral para el año $1\left(\mathrm{R}^{2}=0.99\right)$ (Figura $\left.1 \mathrm{C}\right)$. Algo similar se obtuvo al usar el modelo del año 1 para predecir el desarrollo floral en el año $2\left(R^{2}=0.97\right)$ (Figura 1D).

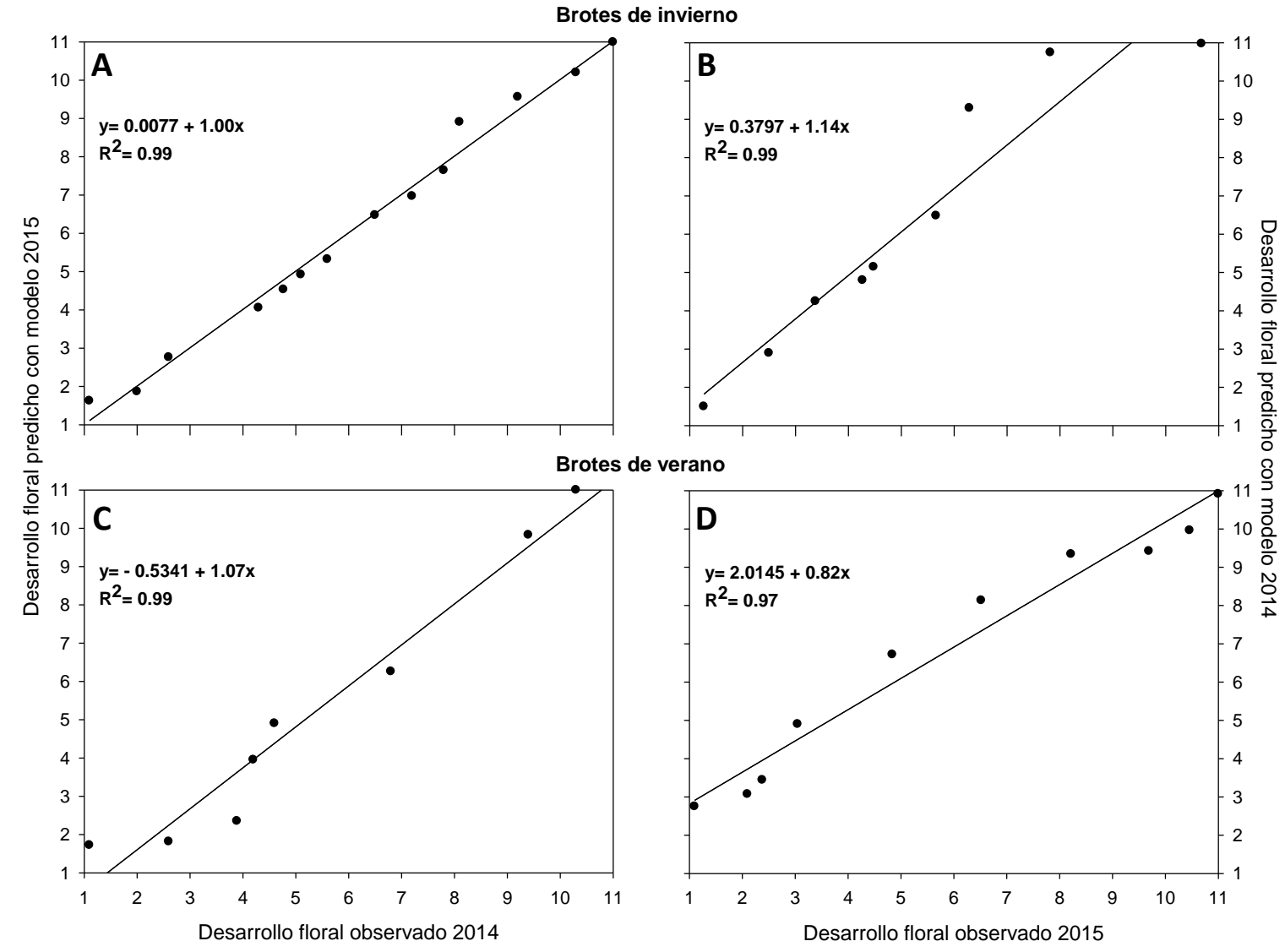

Figura 1. Ajuste de valores entre el estado de desarrollo floral observado en el año 1 (2014) vs. el desarrollo floral predicho obtenido para el año 2 (2015) y viceversa en brotes del flujo de invierno con los modelos DFA $\leq 17$ (A y B) y de verano DFA $\leq 15^{\circ} \mathrm{C}$ (C y D). 


\section{Nuevos modelos generados con datos de dos años}

Los modelos de predicción para brotes del flujo de invierno y de verano del año 1 fueron capaces de predecir el desarrollo floral del año 2 y viceversa. Ante esto, se integró un solo conjunto de datos con información de dos años para cada tipo de brote, obteniéndose dos nuevos modelos de predicción: invierno $\leq 17{ }^{\circ} \mathrm{C}$ y verano $\leq 15^{\circ} \mathrm{C}$ (Cuadro 5). Estos modelos fueron incorporados a una aplicación (www.cesix.inifap.gob.mx/frutalestropicales/desarrollofloralmendezjalisco.php) la cual muestra el desarrollo floral de cada flujo vegetativo del aguacate 'Méndez'. De esta manera es posible usar esta información para la mejor programación de las actividades de manejo del huerto que pudiesen inhibir o disminuir la intensidad de floración, o bien planear la introducción al huerto de insectos polinizadores. Otro uso de los modelos de predicción del desarrollo floral es apoyar a estudiantes, técnicos e investigadores involucrados en el cultivo del aguacate 'Méndez'.

Cuadro 5. Modelos de predicción del desarrollo floral del aguacate 'Méndez' obtenidos a partir de datos de dos años (2014 y 2015) y basados en DFA con temperaturas $\leq 17{ }^{\circ} \mathrm{C}$ (brotes de invierno) y $\leq 15^{\circ} \mathrm{C}$ (brotes de verano).

\begin{tabular}{|c|c|c|c|c|c|c|c|c|c|c|}
\hline $\begin{array}{c}\text { Tempe- } \\
\text { ratura }\end{array}$ & Bo & $\mathrm{B}_{1}$ & $\mathrm{~B}_{2}$ & $\mathrm{~B}_{3}$ & $\mathrm{~B}_{4}$ & $\mathrm{~B}_{5}$ & $\mathrm{~B}_{6}$ & $\mathrm{R}^{2}$ & $\mathrm{Cp}$ & CME \\
\hline \multicolumn{11}{|c|}{ Parámetros (brotes de invierno) } \\
\hline$\leq 17^{\circ} \mathrm{C}$ & 1.2436 & -0.0129 & & 0.000038 & $-5.24 \mathrm{E}-07$ & 2.6E-09 & $-4.3 \mathrm{E}-12$ & 0.69 & 3.98 & 3.43 \\
\hline \multicolumn{11}{|c|}{ Parámetros (brotes de verano) } \\
\hline$\leq 15^{\circ} \mathrm{C}$ & 1.79412 & -0.0399 & 0.0055 & -0.00014 & 0.0000015 & $-7.77 \mathrm{E}-09$ & $1.43 \mathrm{E}-11$ & 0.85 & 5.04 & 2.14 \\
\hline
\end{tabular}

Registro de temperaturas. Durante el periodo de estudio, las temperaturas mínimas más bajas se presentaron en el primer trimestre 2016 (Figura 2). En la semana seis y 11 se registraron 6 y $5{ }^{\circ} \mathrm{C}$, respectivamente. Por otra parte, en el 2014 entre la semana 25 y 29 (última de junio y julio, respectivamente) las temperaturas mínimas alcanzaron en promedio $21.8^{\circ} \mathrm{C}$.

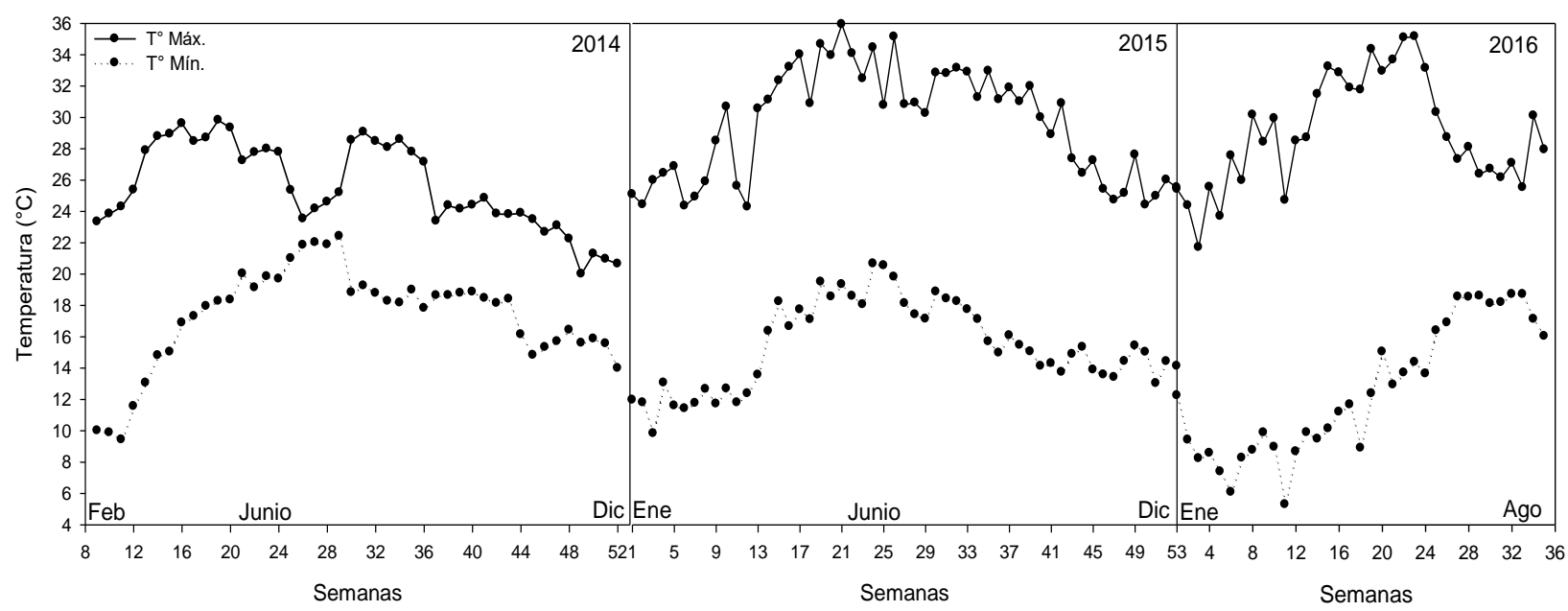

Figura 2. Temperaturas máximas y mínimas del aire registradas de febrero 2014 a agosto 2016. Promedio semanal de datos de los huertos "Colorín 1" y "Ocote cuate 2". 


\section{Conclusiones}

En esta investigación por primera vez fue modelado matemáticamente el desarrollo floral de yemas apicales de los flujos vegetativos de invierno y verano del aguacate 'Méndez' a temperaturas $\leq 17(126 \mathrm{DFA})$ y $15^{\circ} \mathrm{C}(138 \mathrm{DFA})$ respectivamente. Las diferencias entre estas temperaturas y las encontradas para otros cultivares de aguacate, incluyendo a Hass, muestran la plasticidad genética del aguacate para adaptarse a los diferentes ambientes en los que se le cultiva y la irrelevancia de establecer un valor único o crítico de temperatura asociada al desarrollo floral ya que el "frío" necesario para iniciar y completar un ciclo de desarrollo floral depende de las temperaturas que ocurren en cada región productora.

\section{Literatura citada}

Buttrose, M. S. and Alexander, D. M. 1978. Promotion of floral initiation in 'Fuerte' avocado by low temperature and short daylength. Netherlands. Scientia Hort. 8:213-217.

Chaikiattiyos, S.; Menzel, C. M. and Rasmussen, T. S. 1994. Floral induction in tropical fruit trees: effects of temperature and water supply. United Kingdom. J. Hort. Sci. Biotech. 69:397-415.

Davenport, T. L. 1982. Avocado growth and development. Proc. Fla. State Hort. Soc. 95:92-96.

Draper, N. R. and Smith, H. 1981. Applied regression analysis. Ed. John Wiley \& Sons, Inc. Second Edition. New York, USA. 709 p.

García, A. E. 1998. Comisión Nacional para el estudio de la Biodiversidad (CONABIO). Cartas de clima de la República Mexicana. Modificaciones al sistema de clasificación de Köppen. Escala 1:1000 000 México. Shapefile. http://www.igeograf.unam.mx/sigg/ utilidades/docs/pdfs/publicaciones/geo_siglo21/serie_lib/modific_al_sis.pdf.

López-Cruz, I. L.; Ramírez, A. A. y Rojano, A. A. 2005. Modelos matemáticos de hortalizas en invernadero: trascendiendo la contemplación de la dinámica de cultivos. México. Rev. Chapingo Ser. Hort. 11(2):257-267.

Nevin, J. M. and Lovatt, C. J. 1989. Changes in starch and ammonia metabolism during low temperature stress-induced flowering in Hass avocado-A preliminary report. Republic of South African. South African Avocado Growers' Assn. Yrbk. 12:21-25.

Rocha, A. J. L.; Salazar, G. S.; Bárcenas, O. A. E.; González, D. I. J. L. y Cossio, V. L. E. 2011. Fenología del aguacate 'Hass' en Michoacán. México. Rev. Mex. Cienc. Agríc. 2(3):303-316.

Salazar-García, S. 2002. Nutrición de aguacate, principios y aplicaciones. Instituto Nacional de Investigación Forestales, Agrícolas y Pecuarias (INIFAP) en asociación con el Instituto de la Potasa y el Fósforo (INPOFOS). Querétaro, Querétaro, México. 165 p.

Salazar-García, S.; Lord, E. M. and Lovatt, C. J. 1998. Inflorescence and flower development of the 'Hass' avocado (Persea americana Mill.) during "on" and "off" crop years. USA. J. Amer. Soc. Hort. Sci. 123:537-544.

Salazar-García, S.; Lord, E. M. and Lovatt, C. J. 1999. Inflorescence development of the 'Hass' avocado: commitment to flowering. USA. J. Amer. Soc. Hort. Sci. 124:478-482.

Salazar-García, S.; Cossio, V. L. E.; Lovatt, C. J.; González, D. I. J. L. and Pérez, B. M. H. 2006. Crop load affects vegetative growth flushes and shoot age influences irreversible commitment to flowering of 'Hass' avocado. USA. HortSci. 41:1541-1546. 
Salazar-García, S.; Cossio, V. L. E.; González, D. I. J. L.and Lovatt, C. J. 2007. Desarrollo floral del aguacate 'Hass' en clima semicálido. II. Generación y validación de modelos de predicción del desarrollo floral. México. Rev. Chapingo Ser. Hort. 13:93-101.

Salazar-García, S.; Cossio, V. L. E. y González, D. I. J. L. 2009. Validación de modelos de predicción del desarrollo floral del aguacate 'Hass' desarrollados para Nayarit, en varios climas de Michoacán. México. Rev. Chapingo Ser. Hort. 15(3):281-288.

SAS. 2011. Statistical Analysis System. User's Guide: Mathetmatical Programming. Version 9.3. Cary, NC. USA. 